\title{
An investigation into exclusive breast feeding practices in rural Bangladeshi women
}

\author{
S. R. Haque and R. Ash \\ H.D. McCarthy London Metropolitan University, London, N7 8DB, UK
}

In Bangladesh breastfeeding culture is very strong but the practices of prelacteal feeding, additional feeding and early weaning makes exclusive breastfeeding for 6 months as recommended by WHO almost non-existent ${ }^{(1,2,3,4)}$. The aim of this observational study was to characterize breast feeding, additional feeding and weaning practices in a sample of rural Bangladeshi women. The focus was on the timing of the introduction additional and complementary foods and the association of these with diarrheal disease and the nutritional status of the infants.

201 mothers with infants aged 0-12 months, attending the 'Expanded Program on Immunization' (EPI) at Narayanganj General Hospital, near Dhaka between March and April 2012 were interviewed by nurses using a questionnaire developed from the existing literature on infant weaning practices in S.Asia. The mothers' had a mean age of 24.9 years and $87.6 \%$ were literate. $95.5 \%$ were housewives; $56 \%$ come from joint family and 44\% from a nuclear family background. The mean age of the infants was 6 months and $54 \%$ were male and $46 \%$ female. $54.7 \%$ of infants were in the normal range of weight for age; $21.4 \%$ were under weight and $23 \%$ were severely underweight; $1 \%$ was overweight ${ }^{(5)}$.

Only $6.7 \%$ of the infants were exclusively breast fed for 6 months. Almost half, $47 \%$ of the infants had received additional or weaning foods by 2 months of age and the remaining $46 \cdot 2 \%$ by 5 months. $87.7 \%$ mothers said they had started additional feeds or weaning because of insufficient breast milk and or an increased demand from the baby; these associations were significant. Most (68.5\%) of these infants received powder milk or diluted cow's milk as an additional feed. Reported diarrhoea was significantly associated with both powder milk $(p<0.05)$ and diluted cow's milk $(p<0 \cdot 001)$. Additional feeding below 2 months was also significantly associated with diarrhoea $(p<0.05)$, with $65.8 \%$ of the infants affected. Nearly all, $(93 \%)$ of the severely underweight infants had been fed powder milk or diluted cow's milk before six months as well as most of $(80.8 \%)$ the underweight children. $69 \%$ of infants given Shuji (semolina mixed with milk and sugar) as a complementary feed were of normal weight for age. In Bangladesh, several other studies have shown that the incidence of diarrhoea is higher among infants given additional feeding before 6 months compared to those exclusively breast fed ${ }^{(6)}$.

Our data demonstrates that additional feeding before 6 months with powder milk and/or diluted cow's milk is significantly associated with diarrheal disease and had a major impact on nutritional status of these infants. We will be conducting an intervention to reduce this practice of additional feeding before 6 months.

S.H.R. acknowledges PhD grant funding by Bangladesh Science and ICT Project of Bangladesh Govt.

1. World Health Organization (2011). Up to what age can a baby stay well- nourished just by being breast feed?

2. Khan MAS., Hossain MM., Razzak A et al. (2008) Factors of weaning practices by mothers on children: A hospital based study. Orion Medical $J$, 2008 May; 30: 561-564.

3. Greiner T (1997). Breast feeding in Bangladesh: A Review of the Literature, Bangladesh J of Nutr, Vol.10.Nos 1 \& 2, June, 1997.

4. Giashuddin MS, Kabir M, Rahman A et al. (2003) Exclusive breastfeeding and nutritional status in Bangladesh. Indian J Pediatr. 2003 Jun; 70(6), $471-5$.

5. World Health Organization (2008). Child Growth Standards, Interpreting growth indicators.

6. Mitra SN, Ali MN, Islam S, Cross AR et al. (1994). Bangladesh Demographic and Health Survey, 1993-1994. National Institute of Population Research and Training, Dhaka, Bangladesh. 